\title{
Biosynthesis, Characterization and Antibacterial Capability of Silver and Copper Nanoparticles Using Aqueous Leaf Extract of Salacia chinensis L
} Jaykumar J Chavan ${ }^{1,2 *}$ and Dhanaji M Ghadage ${ }^{1}$

${ }^{1}$ Department of Botany, Yashavantrao Chavan Institute of Science, Satara, India

${ }^{2}$ Department of Biotechnology, Yashavantrao Chavan Institute of Science, Satara, India

\begin{abstract}
Salacia chinensis $\mathrm{L}$. is highly valued taxa having several medicinal implications. The study aimed to synthesize and characterize the silver (AgNPs) and copper nanoparticles (CuNPs) using the aqueous leaf extract of $S$. chinensis and their test against pathogenic bacteria. The silver and copper nanoparticles were synthesized using different concentrations of aqueous $\mathrm{AgNO}_{3}$ and $\mathrm{CuNO}_{3}$ solutions; however $4 \mathrm{mM}$ was found optimal concentration. The synthesized nanoparticles were characterized using UV-Vis spectroscopy; scanning electron microscopy and the antibacterial capability of nanoparticles were evaluated using agar well diffusion method. The AgNPs and CuNPs were formed within an hour of the reaction and showed maximum UV-Vis absorption at $445 \mathrm{~nm}$ and $570 \mathrm{~nm}$ respectively. SEM analysis revealed the size $(50-200 \mathrm{~nm})$ and diverse shape (spherical, road) of the synthesized nanoparticles. The efficiency of synthesized AgNPs and CuNPs against the bacterial species viz. Escherichia coli, Bacillus subtilis, Staphylococcus aureus and Pseudomonas averogenosa validated the action of these nanoparticles against these infectious microbes. The study illustrated the capacity of leaves of $S$. chinensis towards the synthesis of silver and copper nanomaterial and AgNPs were found with enhanced antibacterial capability against test microbes.
\end{abstract}

Keywords: Antibacterial capability; Copper nanoparticles; Leaf extract; Salacia chinensis; SEM; Silver nanoparticles

\section{Introduction}

Saptarangi (Salacia chinensis L.) is highly valued medicinal plant of family Hippocrateaceae which is commonly known as Saptarangi and Saptachakra in the Ayurvedic system of traditional medicines [1]. Different plant parts of $S$. chinensis are compelling source of active phytoconstituents like phenolics, flavonoids and good natural source of antioxidants [2-4]. The roots also showed the presence of antidiabetic and anticancerous principles like salacinol, kotalanol, and mangiferin [5-8]. The plant also functions as astringent, abortifacient carminative, blood tonic, cardio-tonic, in amenorrhea and dysmenorrhoea, $[1,5,9]$ and used in treatments of various ailments and diseases [10]. Various extracts of S. chinensis also described to possess several pharmacological properties including antimicrobial, antihyperglycemic, anti-inflammatory, immuno-modulatory, anti-mutagenic activities antitumor, anti-HIV and immunomodulatory activities [2,5,11-13].

In recent years, synthesis of metal nanoparticles is a subject of focused research interest due to its wide potential applications in biomedical, optical and electronic fields [14]. The enhanced pharmacological and biomedicinal performance of $S$. chinensis can be achieved with metal nanomaterials. Literature reveals that, the stem of Salacia chinensis has the capability towards synthesis of silver nanoparticles [15]. However, leaves of $S$. chinensis are chief source of many bioactive constituents. Hence in the present study, reducing potential of $S$. chinensis aqueous leaf extract have been exploited for the eco-friendly and bio-utilizable synthesis of silver (AgNPs) and copper nanoparticles (CuNPs). These nanoparticles were characterized by UV-VIS spectroscopy, SEM and the antibacterial properties were also evaluated.

\section{Material and Methods}

\section{Chemicals and materials}

Silver nitrate $\left(\mathrm{AgNO}_{3}\right)$, copper nitrate $\left(\mathrm{CuNO}_{3}\right)$, Nutrient Agar and MacConkeys agar were procured from Hi-Media, India. $S$. chinensis leaves were collected from Amboli locality of the Northern Western Ghats. Bacterial species viz; Bacillus subtilis, Escherichia coli,
Staphylococcus aureus, Pseudomonas averogenosa were obtained from the Department of Microbiology and Biotechnology, Yashavantrao Chavan Institute of Science, Satara.

\section{Preparations of Salacia chinensis leaf extract (SCLE)}

Freshly collected leaves of $S$. chinensis were washed thoroughly under running tap water to remove traces of impurities and were blotted to dry. Shade dried leaves were then powdered using grinder. Ten gram powder was suspended for extraction in $100 \mathrm{ml}$ boiling distilled water for 2 hours on water bath. Initially, the suspension was filtered through muslin cloth and then with Whatmann filter paper 1 to get the crude extract. Final volume of Salacia chinensis leaf extract (SCLE) was adjusted to $100 \mathrm{ml}$ by distilled water and the same was used for synthesis of AgNPs and CuNPs.

\section{Synthesis of silver nanoparticles (AgNPs)}

SCLE extract $(10 \% \mathrm{w} / \mathrm{v})$ were added to $4 \mathrm{mM}$ aqueous $\mathrm{AgNO}_{3}$ solution in 1:4 concentrations. The reaction was carried out at room temperature ranging from 25 to $30^{\circ} \mathrm{C}$ for 60 minutes. Synthesis of AgNPs was established by colour change and UV-Visible spectrophometric analysis. AgNPs were separated out by centrifugation and washed with sterile distilled water. Purified AgNP pellets were kept for oven drying at $60^{\circ} \mathrm{C}$ for $24 \mathrm{~h}$ which were then scrapped out and stored.

\section{Synthesis of copper nanoparticles (CuNPs)}

CuNPs were synthesized by using 10\% SCLE and the $4 \mathrm{mM}$

*Corresponding author: Jaykumar J Chavan, Department of Botany, Yashavantrao Chavan Institute of Science, Satara 415001, India, Tel: 9102162234392; Fax: 9102162234392; E-mail: jaychavansu@gmail.com

Received: December 11, 2017; Accepted: February 21, 2018; Published: February 26, 2018

Citation: Chavan JJ, Ghadage DM (2018) Biosynthesis, Characterization and Antibacterial Capability of Silver and Copper Nanoparticles Using Aqueous Leaf Extract of Salacia chinensis L. J Nanomed Nanotechnol 9: 484 doi: 10.4172/2157 7439.1000484

Copyright: (c) 2018 Chavan JJ, et al. This is an open-access article distributed under the terms of the Creative Commons Attribution License, which permits unrestricted use, distribution, and reproduction in any medium, provided the original author and source are credited. 
aqueous $\mathrm{CuNO}_{3}$ solution in a $1: 4$ ratio at room temperature (25 to $30^{\circ} \mathrm{C}$ ) for $60 \mathrm{~min}$. Change in colour indicated synthesis of CuNPs. The reaction mixture was centrifuged and CuNPs were separated out. After washing with sterile distilled water, purified pellets were transferred to petri plate and were kept for oven drying at $60^{\circ} \mathrm{C}$ for $24 \mathrm{~h}$. The dried CuNPs were scrapped out for the further study.

\section{Characterization of Nanoparticles}

The UV-Vis spectrometric scan was carried out on Systronics-119 to monitor the bioreduction of AgNPs and CuNPs at room temperature at the resolution of $1 \mathrm{~nm}$ between 300 to $800 \mathrm{~nm}$ ranges as a function of time. Reaction mixture $(2 \mathrm{ml})$ was analyzed every time from initiation of reaction to 60 minutes at time intervals of 10 minutes. The surface morphology and size range was recorded by scanning electron microscopy (JEOL JSM 6360 with an accelerating voltage $20 \mathrm{kV}$ ). SEM analysis was performed by the previously described procedure [15]. A drop of AgNPs and CUNPs colloidal suspension was deposited on carbon grid and dried under low vacuum (10-130 pa). The samples were scanned at magnification of 3000x and the spot was fixed and was analyzed by EDX system to confirm the presence of silver and copper.

The antimicrobial activity of AgNPs and CuNPs were evaluated by using the agar well diffusion method against the pathogenic bacteria including gram positive viz. Bacillus subtilis, Staphylococcus aureus and gram negative viz. Escherichia coli, Pseudomonas averogenosa [16]. The bacterial cultures were maintained on Nutrient Agar and MacConkeys agar slants. The microorganism was activated by inoculating a loopful of the strain in the nutrient broth $(25 \mathrm{ml})$ and incubated at room temperature. Inoculum $(0.5 \mathrm{ml})$ was added to the agar plates. For the agar well diffusion, a well was made in the seeded plates with the help of a cup-borer $(8.5 \mathrm{~mm})$ in the medium. Freshly prepared AgNPs samples $(100 \mu \mathrm{L})$ and CuNPs $(100 \mu \mathrm{L})$ were added into the wells. The SCLE extract $(100 \mu \mathrm{L})$ used as a negative control while streptomycin was used as positive control. The samples were kept for incubation for 24 $\mathrm{h}$ at $37^{\circ} \mathrm{C}$. A zone of inhibition was observed around the well after the incubation period and the observations were recorded.

\section{Results and Discussion}

\section{Synthesis of silver (AgNPs) and copper (CuNPs) nanoparticles}

Silver and copper nanoparticles were synthesized by addition of S. chinensis leaf extract to the solutions of silver nitrate and copper nitrate. The leaves are capable to synthesize the silver and copper nanoparticle. The synthesis of AgNPs were confirmed based on visual observation of colour change from colourless to brown and synthesis of CuNPs was confirmed as of colourless to reddish brown
(Figure 1A-1E). The synthesized nanoparticles were reduced easily and were found stable. This might me due to the presence of bioactive constituents including kotalanol, salacinol and mangiferin in the leaves of S. chinensis. Similarly, stable metal nanoparticles viz. silver, copper and gold nanoparticles were synthesized from leaves of Artemisia nilagirica [17] and Terminalia arjuna [18]. In the present study, the AgNPs and CuNPs were successfully separated and purified by repeated centrifugation. The dispersal in to the deionized water was also confirmed the purity of synthesized nanoparticles.

\section{Characterization of AgNPs and CuNPs}

In the present study, along with of SCLE, $\mathrm{AgNO}_{3}$ extract showed absorbance peak at $445 \mathrm{~nm}$ which confirmed the synthesis of AgNPs. However, an individual aqueous extracts of SCLE, $\mathrm{AgNO}_{3}$ and $\mathrm{CuNO}_{3}$ did not showe any peak (Figure 2A). Synthesis of AgNPs confirmed by absorbance peak at $445 \mathrm{~nm}$ (Figure $2 \mathrm{~B}$ ) are in line with previous report as the $\lambda$ max values of AgNPs were found in the visible range of 400-500 nm [19]. However, the larger particle size might shift in the plasmon band for higher wavelength [20]. Formation of CuNPs was confirmed by UV spectroscopy which showed a characteristic peak at $630 \mathrm{~nm}$ (Figure 2C).

Scanning electron microscopy provided the platform for studying the size and shape of the metal nanoparticles. SEM microphotographs of the AgNPs and CuNPs obtained by the reduction of aqueous leaf extract of $S$. chinensis revealed the nanoparticles differed in size as well as shape (Figure $3 \mathrm{~A}$ and $3 \mathrm{~B}$ ). The resulting silver nanoparticles were in the size range of 50-200 $\mathrm{nm}$. Morphologically polymorphic AgNPs are triangular, hexagonal, deformed spherical and rod shaped, however the CuNPs were tubular and rod shaped. Both the nanoparticles showed the aggregation and nanocluster might be due to the dryness stimulated by evaporation of solvent during the reaction period. Synthesis in group or bunch may lead to decrease in their biological activities [21].

\section{Antibacterial activity of AgNPs and CuNPs}

AgNPs and CuNPs are recognized as the broad spectrum antimicrobial agents with its wide application potential in medical and agricultural field $[22,23]$. The antibacterial results obtained for $S$. chinensis leaf derived AgNPs and CuNPs against test organisms are represented in Figure 4A and 4B. The zone of inhibition observed after 16-18 hours of activity. The present study revealed that, Escherichia coli, Bacillus subtilis are more susceptible to AgNPs while Pseudomonas averogenosa and Streptococcus aureus showed minimal level of susceptibility (Figure 5). In the present study, AgNPs have the superior antibacterial capability against tested bacterial strains as compared to CuNPs. This might be the synthesis of CuNPs in the clusters or in

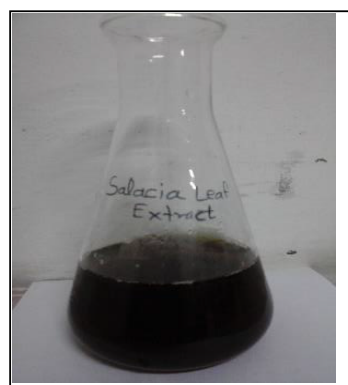

Leaf Extract

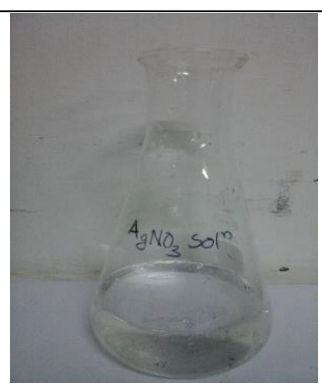

$\mathrm{AgNO}_{3}$ Solution

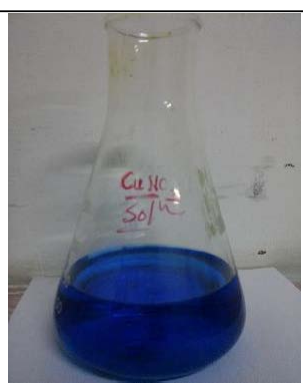

$\mathrm{CuNO}_{3}$ Solution

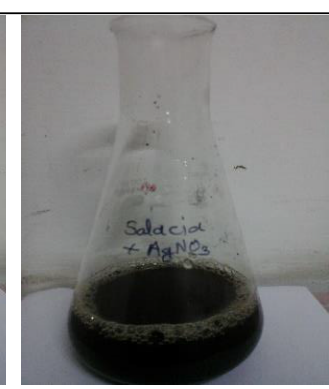

Leaf Extract $+\mathrm{AgNO}_{3}$

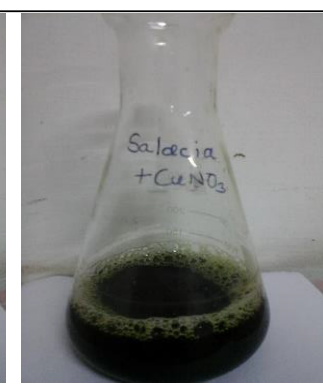

Leaf Extract $+\mathrm{CuNO}_{3}$

Figure 1: Biosynthesis of AgNPs and CuNPs from aqueous leaf extracts of $S$. chinensis 
Citation: Chavan JJ, Ghadage DM (2018) Biosynthesis, Characterization and Antibacterial Capability of Silver and Copper Nanoparticles Using Aqueous Leaf Extract of Salacia chinensis L. J Nanomed Nanotechnol 9: 484. doi: 10.4172/2157-7439.1000484

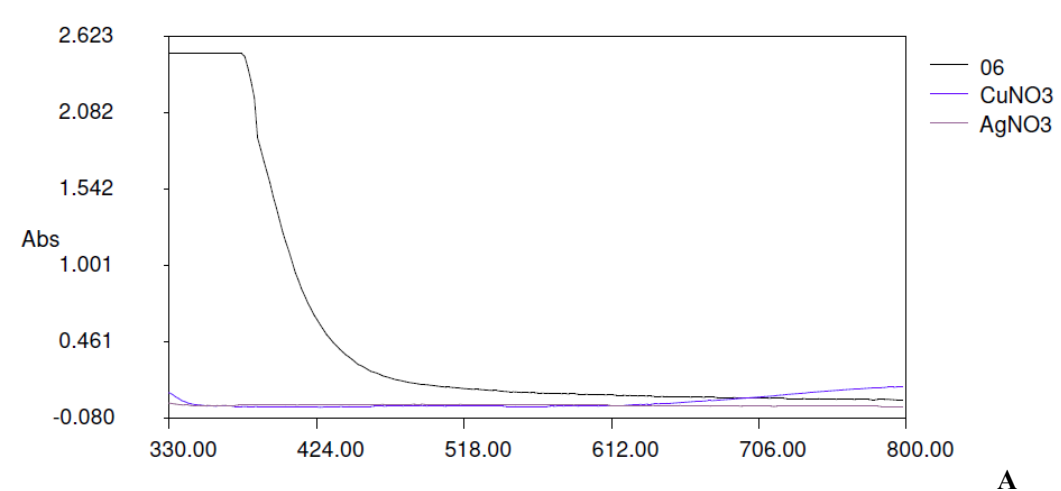

Base Graph : — $\quad$ _ $\quad$ _

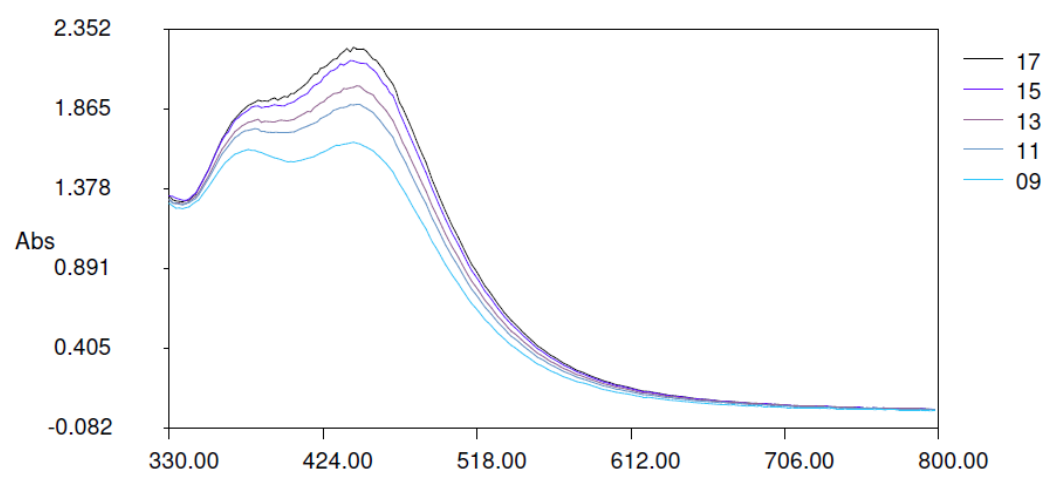

Base Graph : $\quad$

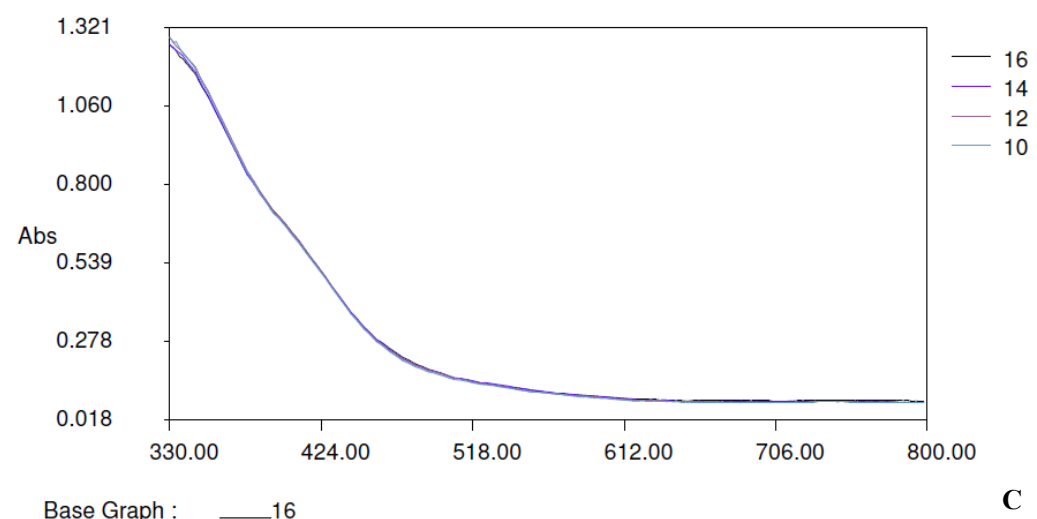

Figure 2: UV-visible profiles. A: Aqueous leaf extract, $\mathrm{AgNO}_{3}$ and $\mathrm{CuNO}_{3}$; B: Synthesized AgNPs and C: Synthesized CuNPs.

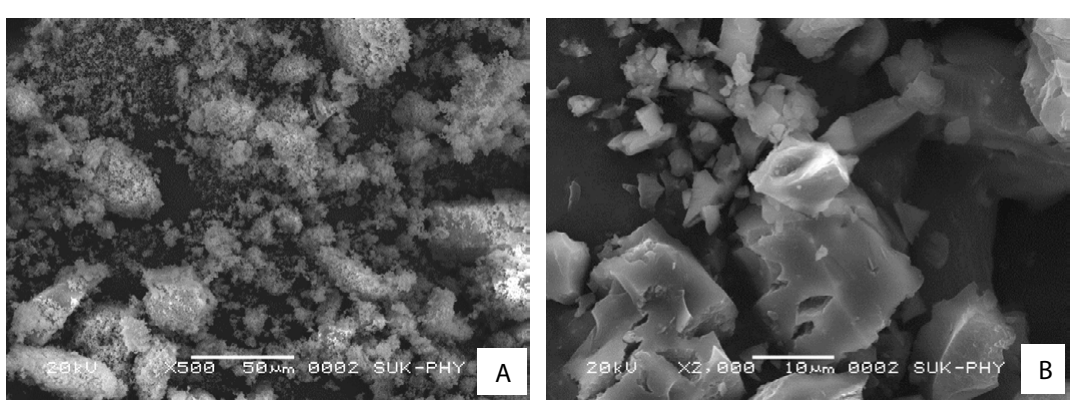

Figure 3: Scanning electron micrograph of synthesized nanoparticles. A: AgNPs and B: CuNPs. 
Citation: Chavan JJ, Ghadage DM (2018) Biosynthesis, Characterization and Antibacterial Capability of Silver and Copper Nanoparticles Using Aqueous Leaf Extract of Salacia chinensis L. J Nanomed Nanotechnol 9: 484. doi: 10.4172/2157-7439.1000484
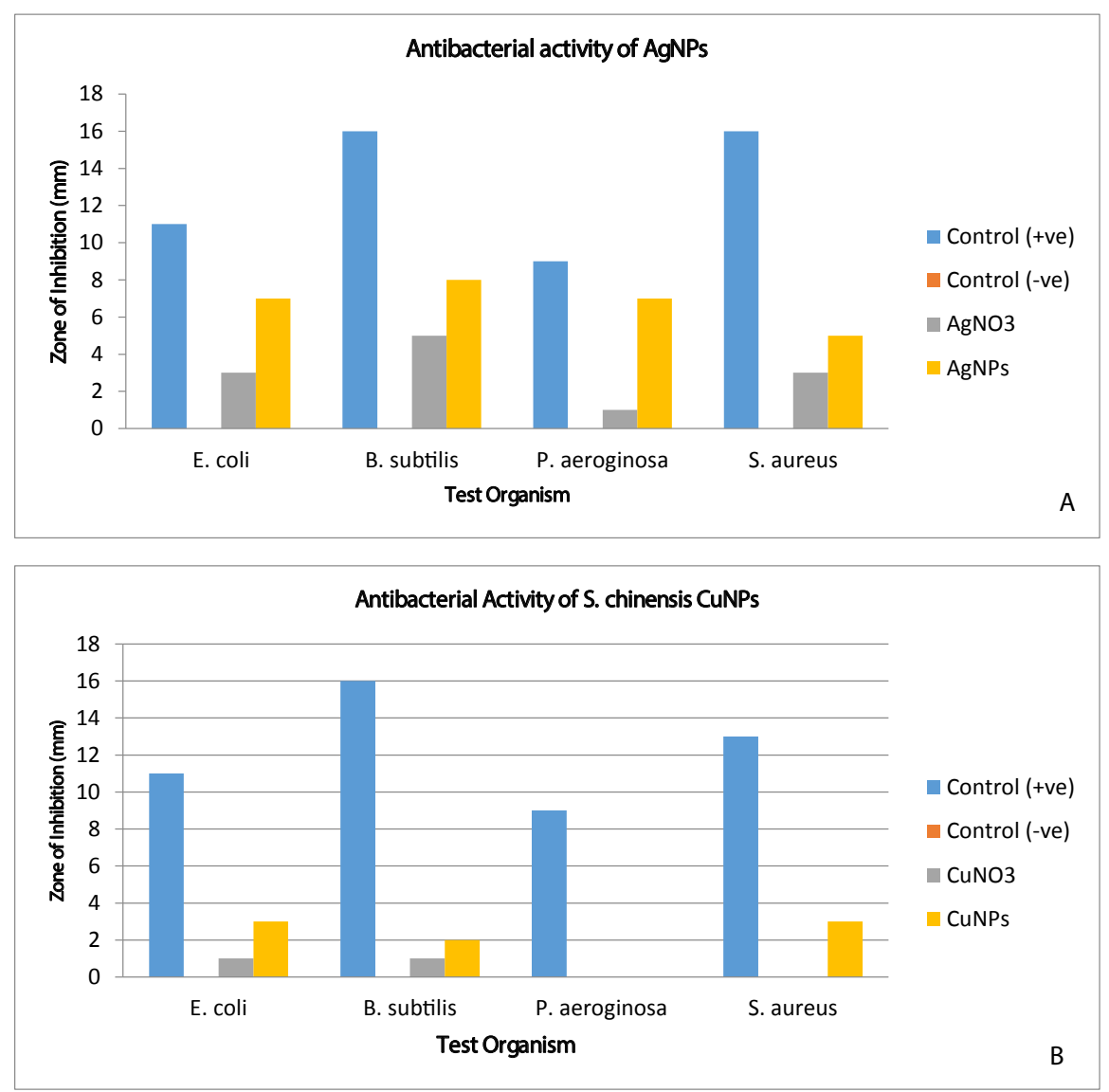

Figure 4: Effect of nanoparticles on pathogenic bacteria. A: AgNPs and B: CuNPs.

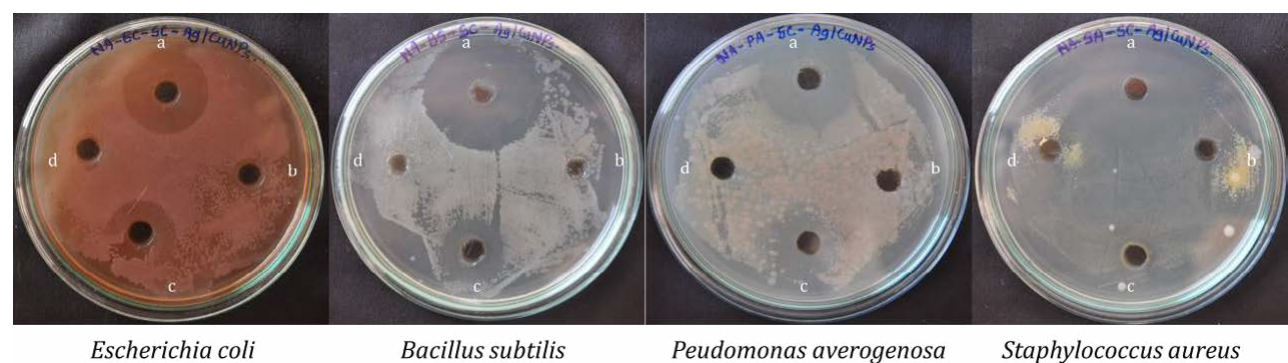

a: +ve control (Streptomycin), b: S. chinensis aq. leaf extract, c: AgNPs d: CuNPs

Figure 5: Antibacterial activity assay against Gram +ve and Gram -ve bacteria depicting zone of inhibitions.

aggregates instead of singular synthesis. The findings are competent with the previous study which confirmed that silver nanoparticles are effective against human pathogens and as antimicrobial agents $[15,24,25]$. The metal nanoparticles attaches to surfaces of cell membranes as granules [26] troubling the permeability of membranes and respiratory functions, with dysfunction of metabolic pathways. It also inhibits the cell division by damaging inner cell organelles and DNA [27].

\section{Conclusion}

Eco-friendly and cost-effective procedure is developed for biosynthesis of silver and copper nanoparticles from aqueous leaf extract of $S$. chinensis. The leaves showed the bioreduction potential towards $\mathrm{AgNO}_{3}$ and $\mathrm{CuNO}_{3}$ to produce AgNPs and CuNPs. Owing to its antibacterial properties, the AgNPs showed its efficiency towards different gram positive and gram-negative bacterial strains which suggest that silver metal in nanoparticles range could be effectively used as an antibacterial agent. Comparatively, AgNPs were more efficient against tested microbes than the CuNPs. The bio-utilizable size of AgNPs makes it useful against development of antimicrobial drugs, in medical devices and also in agricultural field.

\section{Acknowledgement}

Authors are also thankful to Head, Department of Botany and the Principal, Yashavantrao Chavan Institute of Science, Satara for providing necessary 
Citation: Chavan JJ, Ghadage DM (2018) Biosynthesis, Characterization and Antibacterial Capability of Silver and Copper Nanoparticles Using Aqueous Leaf Extract of Salacia chinensis L. J Nanomed Nanotechnol 9: 484. doi: 10.4172/2157-7439.1000484

laboratory facilities. We also indebted to Mr. Pavan Hande, Mr. Abhijit Kadam and Mr. Sujit Patil for technical help. Authors are thankful to Department of Science and Technology (DST), Govt. of India New Delhi for financial support (Sanction No. SB/ FT/LS-259 dated 02 May, 2013). Support from the Research Advisory Committee, Y. C. Institute of Science, Satara is also gratefully acknowledged.

\section{Conflict of Interest}

The authors declared no conflicts of interest.

\section{References}

1. Govindaraj Y, Melanaphuru V, Agrahari V, Gupta S, Nema RK (2009) Genotoxicity studies of mangiferin isolated from Salacia chinensis Linn. Acad J Plant Sci 2: 199-204.

2. Chavan JJ, Jagtap UB, Gaikwad NB, Dixit GB, Bapat VA (2012) Total phenolics, flavonoids and antioxidant activity of Saptarangi (Salacia chinensis L.) fruit pulp. J Plant Biochem Biotechnol 22: 409-413.

3. Majid BN, Kini KR, Prakash HS, Geetha N (2016) Phytomorphology, phytochemistry and pharmacological activities of Salacia chinensis L., an endangered antidiabetic medicinal plant: a comprehensive review. Int J Agricult Biosci 5: 1-7.

4. Ghadage DM, Kshirsagar PR, Pai SR, Chavan JJ (2017) Extraction efficiency, phytochemical profiles and antioxidative properties of different parts of Saptarangi(Salacia chinensis L.) - an important underutilized plant. Biochem Biophy Rep 12: 79-90.

5. Yoshikawa M, Nishida N, Shimoda H, Takada M, Kawahara Y, et al. (2011) Polyphenol constituents from Salacia species: quantitative analysis of mangiferin with alpha-glucosidase and aldose reductase inhibitory activities. Yakugaku Zasshi 121: 371-378.

6. Chavan JJ, Ghadage DM, Bhoite AS, Umdale SD (2015) Micropropagation, molecular profiling and RP-HPLC determination of mangiferin across various regeneration stages of Saptarangi (Salacia chinensis L.). Ind Crop Prod 76: 1123-1132.

7. Chavan JJ, Ghadage DM, Kshirsagar PR, Kudale SS (2015) Optimization of extraction techniques and RP-HPLC analysis of antidiabetic and anticancer drug mangiferin from roots of 'Saptarangi' (Salacia chinensis L.). J Liq Chromatograp Rel Technol. 38: 963-969.

8. Wang RR, Gao YD, Ma CH, Zhang XJ, Huang CG, et al. (2011) Mangiferin an anti-HIV-1 agent targeting protease and effective against resistant strains. Molecules 16: 4264-4277.

9. Matsuda H, Morikawa T, Yoshikawa M (2002) Anti-diabetogenic constituents from several natural medicines. Pure Appl Chem 74: 1301-1308.

10. Li Y, Huang TH, Yamahara J (2002) Salacia root, a unique Ayurvedic medicine, meets multiple targets in diabetes and obesity. Life Sci 82: 1045-1049.

11. Guha S, Ghosal S, Chattopadhyay U (1996) Antitumor, immunomodulatory and anti-HIV effect of mangiferin, a naturally occurring glucosylxanthone. Chemotherapy 42: 443-451.
12. Zheng MS, Lu ZY (1990) Antiviral effect of mangiferin and isomangiferin on Herpex simplex virus. Chin Med J 103: 160-165.

13. Sikarwar MS, Patil MB (2012) Antihyperlipidemic activity of Salacia chinensis root extracts in triton-induced and atherogenic diet-induced hyperlipidemicrats. Ind J Pharmacol 44: 88-92.

14. Taylor R, Coulombe S, Otanicar T, Phelan P, Gunawan A, et al. (2013) Smal particles, big impacts: A review of the diverse applications of nanofluids. J App Phy. 113: 011301.

15. Jadhav KV, Dhamecha DL, Dalvi BR, Patil MB (2015) Green synthesis of silver nanoparticles using Salacia chinensis: characterization and its antibacterial activity. Parti Sci Technol 33: 1-36.

16. Irshad S, Mahmood M, Perveen F (2012) In vitro antibacterial activities of three medicinal plants using agar well diffusion method. Res J Biol 2: 1-8.

17. Vijayakumar M, Priya K, Nancy FT, Noorlidah A, Ahmed ABA (2013) Biosynthesis, characterisation and anti-bacterial effect of plant-mediated silver nanoparticles using Artemisia nilagirica. Ind Crop Prod 41: 235-240.

18. Gopinath K, Venkatesh KS, llangovan R, Sankaranarayanan K, Arumugama A (2013) Green synthesis of gold nanoparticles from leaf extract of Terminalia arjuna, for the enhanced mitotic cell division and pollen germination activity. Ind Crop Prod 50: 737-742.

19. Sastry M, Ahmad A, Khan MI, Kumar R (2004) Nanobiotechnology. Wiley-VCH Weinheim, Germany, 126.

20. Bar H, Bhui DK, Sahoo GP, Sarkar P, De SP, et al. (2009) Green synthesis of Silver nanoparticles using latex of Jatropha curcas. Colloid Surfaces A: Physicochem Eng Asp 339: 134-139.

21. Kvitek L, Panacek A, Soukupova J, Kolar M, Vecerova R, et al. (2008) Effect of surfactants and polymers on stability and antibacterial activity of silver nanoparticles (Nps). J Phy Chem 112: 5825-5834.

22. Kumar VB, Naik H, Girija DK (2012) ZnO nanoparticle as catalyst for efficien green one-pot synthesis of coumarins through knoevenagel condensation. $J$ Chem Sci 123: 1-7.

23. Shende S, Ingale A, Gade A, Rai M (2015) Green synthesis of copper nanoparticles by Citrus medica Linn. (Idilimbu) juice and its antimicrobial activity. World J Microbiol Biotechnol 31: 865-873.

24. Jagtap UB, Bapat VA (2013) Biosynthesis, characterization and antibacteria activity of silver nanoparticles by aqueous Annona squamosa L. leaf extract at room temperature. J Plant Biochem Biotechnol 22: 434-440.

25. Muthukrishnan S, Bhakya S, Senthil Kumar T, Rao MV (2015) Biosynthesis characterization and antibacterial effect of plant mediated silver nanoparticles using Ceropegia thwaitesii - an endemic species. Ind Crop Prod 63: 119-124.

26. Brown TA, Smith DG (1976) Effect of silver nitrate on the growth and ultrastructure of the yeast Cryptococcus albidus. Microbios Let 3: 155-162.

27. Richards R, Odelola ME, Anderson B (1984) Effect of silver on whole cells and spherplast of a silver resistant Pseudomonas arruginosa. Microbios 39 : 151-157. 